Article

\title{
On the Porosity of Cu Coatings Formed in Earth-Based and Space Conditions
}

\author{
Leonid Skatkov $^{1, *}$, Petr Cheremskoy ${ }^{\dagger}$, Valeriy Gomozov ${ }^{2}$, Boris Bayrachny ${ }^{2}$, \\ Gennadiy Tulskiy ${ }^{2}$ and Svetlana Deribo ${ }^{2}$
}

1 Technical Division, Printed Circuit Board “Argo" Ltd., 4/23 Shaul ha-Melekh str., 84797 Beer Sheva, Israel

2 Electrochemistry Department, National Technical University "KhPI", 21 Frunze str., 61002 Kharkov, Ukraine; E-Mails: vg@ecopolymer.com (V.G.); zx47@list.ru (V.G. \& B.B.); tgg@kpi.kharkov.ua (G.T.); sgd.07@mail.ru (S.D.)

$\dagger$ This author deceased on 20 June 2005.

* Author to whom correspondence should be addressed; E-Mail: sf_lskatkov@bezeqint.net; Tel.: +972-8-648-2255; Fax: +972-8-640-1754.

Received: 3 September 2012; in revised form: 14 November 2012 / Accepted: 16 November 2012 / Published: 20 November 2012

\begin{abstract}
This work surveys the structure, substructure and submicroporosity of $\mathrm{Cu}$ metal coatings following condensation both under the conditions of space flight of orbital stations (OS) and under Earth-based conditions. Small-angle X-ray scattering (SAXS) and the method of Ar low-temperature desorption were used for investigation. It has been shown that the condensates deposited in space contain less and relatively equiaxial submicropores (SMPs) distributed more homogeneously than Earth-based disperse films.
\end{abstract}

Keywords: open space; submicropore; $\mathrm{Cu}$; SAXS

\section{Introduction}

This work examines issues related to the structure, substructure and submicroporosity of metal coatings with face-centered cubic lattices of $\mathrm{Cu}$ following condensation both under the conditions of space flight of orbital stations, and under Earth-based conditions. As described by Cheremskoy et al. [1], one major issue in the development of new materials for aerospace engineering 
applications is the porosity problem, which has significant effects on the performance properties of these materials. To control the parameters of porous structures and predict their stability during operation in the presence of multifactorial external inputs (for example, in open space), the origin, mechanisms and kinetics of processes used to develop porous materials must be subjected to systematic testing and data analysis. Previously [2,3], we introduced a theoretical model of pore formation in solids based on thermodynamic considerations. Note that in [4] we conducted an experimental survey of the impact of space on thermostatic metal coatings of black aluminium.

As was shown in [5], after the channels are filled and the entire film has formed a significant amount of macro-, micro- and submicropores (SMPs) remain, which form the following through several different mechanisms: diffusion-vacancy, the shadow effect, non-compact coalescence and joining of the structural elements, uneven growth, gas absorption and micro-contraction. Which mechanism predominates is determined by the physical and chemical conditions of the evaporation and condensation processes. This comparison of the porosity features of space and Earth-based samples is extremely important for the analysis of the impact of orbital station flight conditions on the mechanism of porosity formation.

\section{Results and Discussion}

The surface micro-structures of films produced on Earth and in space are shown in Figures 1-3.

For $\mathrm{Cu}$ films of different thicknesses, the total porosity measured, based on the absorption of monochromatic radiation, reaches several per cent.

Most of the porosity of space and Earth-based samples is constituted of micron-sized macro-pores, which cannot be revealed by SAXS. However, the quantity of SMPs is several times greater than the quantity of macro-pores. The observed small-angle X-ray scattering mostly results from SMP's.

Figure 1 shows SAXS indicatrixes for $\mathrm{Cu}$ films, demonstrating that the SAXS intensity and integral width of the SAXS indicatrixes of Earth-based films are considerably higher than those of samples produced in space.

Figure 1. Small-angle X-ray scattering (SAXS) indicatrix for $\mathrm{Cu}$ coatings precipitated under Earth-based (1) and space (2) conditions.

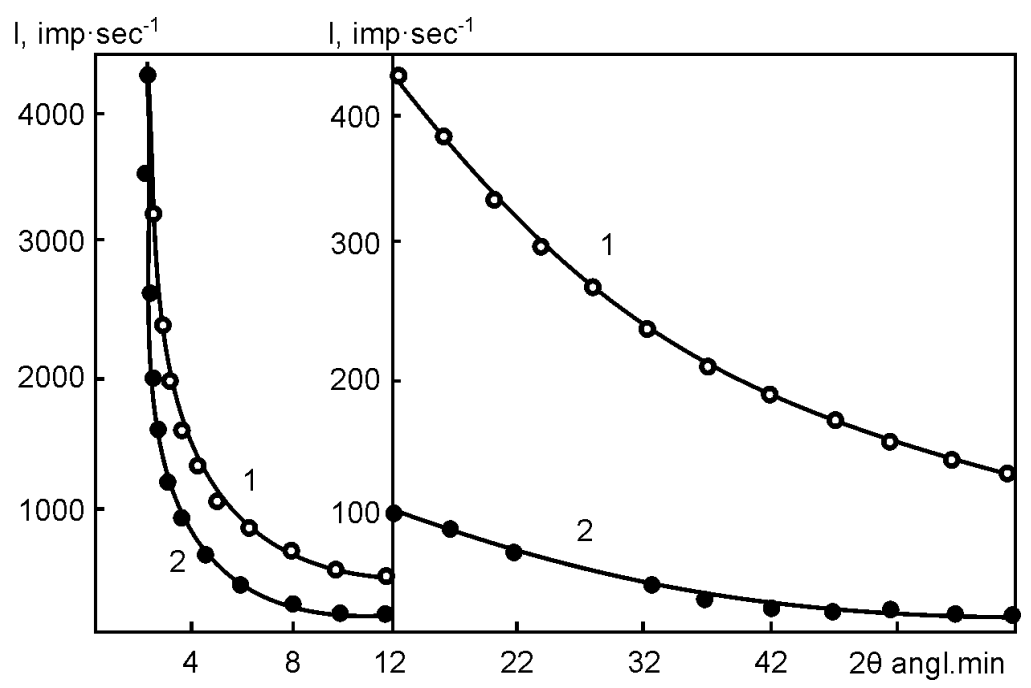


The analysis of SAXS indicatrix asymptotes recorded on an incline reveal that $\mathrm{Cu}$ films mostly contain SMPs, the orientation and anisometry of which are largely determined by aligning the motion of molecular flow during condensation.

The pores of Earth-based samples of $\mathrm{Cu}$ films are in prolate form, and due to their large size, are aligned in a direction close to the molecular flow direction. However, in space, SMP samples are almost equiaxial.

Figure 2. SAXS indicatrix invariant for $\mathrm{Cu}$ coatings precipitated under Earth-based (a) and space (b) conditions.

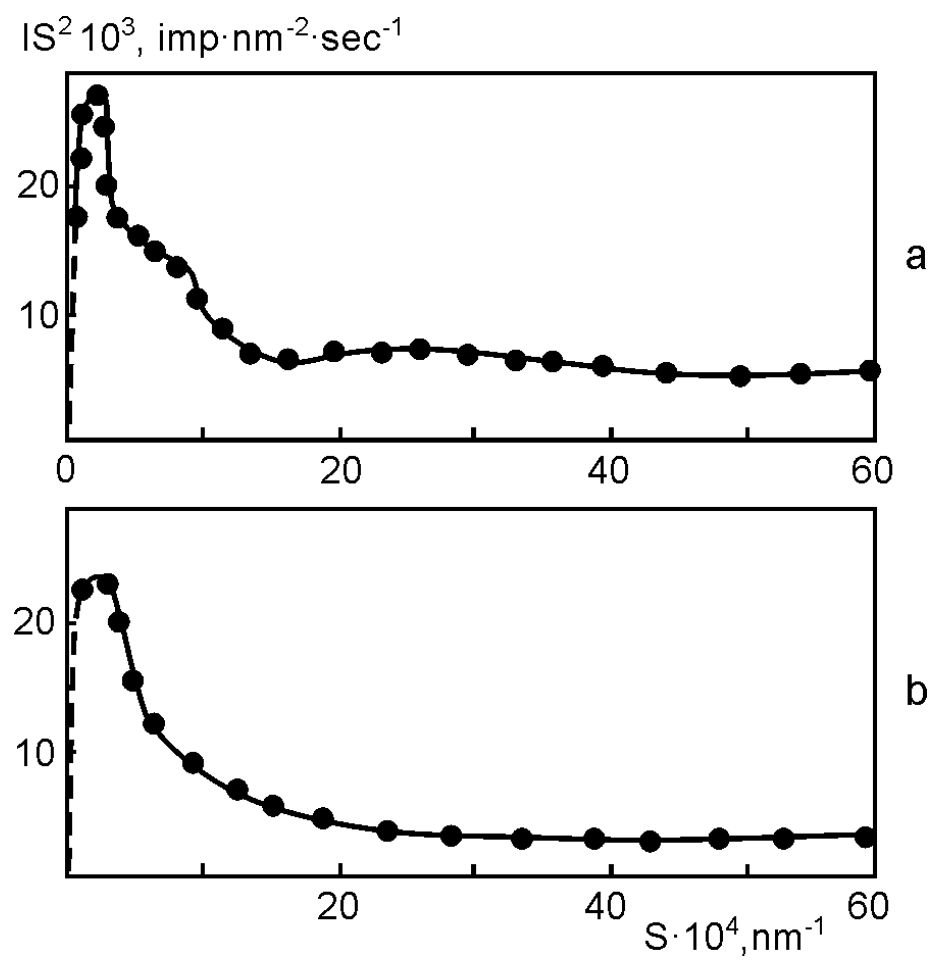

All inspected $\mathrm{Cu}$ space samples display less poly-dispersity of scattering than earth-based samples, which feature an apparent poly-disperse SMP size distribution. This poly-dispersity appears in the form of several maxima on the invariant curves of PMP indicatrixes (Figure 2a). Similar curves for space samples usually have one maximum (Figure $2 b$ ).

At almost the same level of inner porosity, $1.8 \%-2.5 \%$, obtained by the method of monochromatic radiation absorption, the volumetric concentration of SMP in the Earth-based samples is approximately $0.1 \%$ in the metals tested here, compared with a maximum of $0.04 \%$ in the space-based samples. At the same time, the pores in the space-based samples are typically larger than those produced on earth. The mean square and typical sizes of the pores are close to each other, at $30 \mathrm{~nm}$ for Cu Earth-based films, compared with $65 \mathrm{~nm}$ for the corresponding space-based films.

SAXS indicatrixes on Figure 4 are aligned for the same scattering volume as long as the films have different thicknesses. During condensation under Earth-based conditions, the intensity of SAXS films condensed on the stationary support exceeds the intensity of SAXS films condensed on the movable support over the whole range of angular dispersion; this analysis also revealed discrepancies in the angular dispersion of SAXS intensity for both types of films. 
Condensation on a movable support leads to a sharper reduction of SAXS intensity at smaller angles, indicating a difference between the size distribution of scattering irregularities of electron density, which mostly appeared in the form of SMPs or micropores resulting from condensation.

For small film thicknesses, despite the considerable poly-dispersity of SMPs, no SMPs larger than $20 \mathrm{~nm}$ were observed, and this population was symbolically divided into two size fractions $2 R<5 \mathrm{~nm}$ and $5<2 R<40 \mathrm{~nm}$. The total volume and quantity of SMPs in films condensed on the stationary support are substantially higher than in films condensed on the movable support. Key discrepancies are revealed in the fractional contribution of larger SMPs. In both Earth-based and space-based films condensed on the stationary support, the SMP concentration is much higher than in films condensed on the movable support; this difference is smaller for small SMPs.

The films condensed under field conditions on the orbital stations were studied to determine the behaviour of the SAXS intensity, dispersion and volumetric concentration of the SMPs during the growth of the condensate. No substantial discrepancies were observed in SAXS intensity between films of equivalent thickness condensed under Earth-based versus space-based conditions. However, the size distribution of scattering SMPs is observed to be uneven. The intensity rate of SAXS in the films increases along with the condensate thickness (Figure 3b).

Figure 3. SAXS indicatrixes for thin ( $h=0.03-0.25 \mathrm{mkm}) \mathrm{Cu}$ coatings precipitated under Earth-based (a) and space (b) conditions. (a) immobile (1) and mobile (2) substrates, $h=0.175-0.250 \mathrm{mkm}$; (b) immobile substrate: $\bullet, h=0.175 \mathrm{mkm} ; 0,0.08 \mathrm{mkm}$; ., $0.05 \mathrm{mkm} ; \diamond, 0.03 \mathrm{mkm}$.
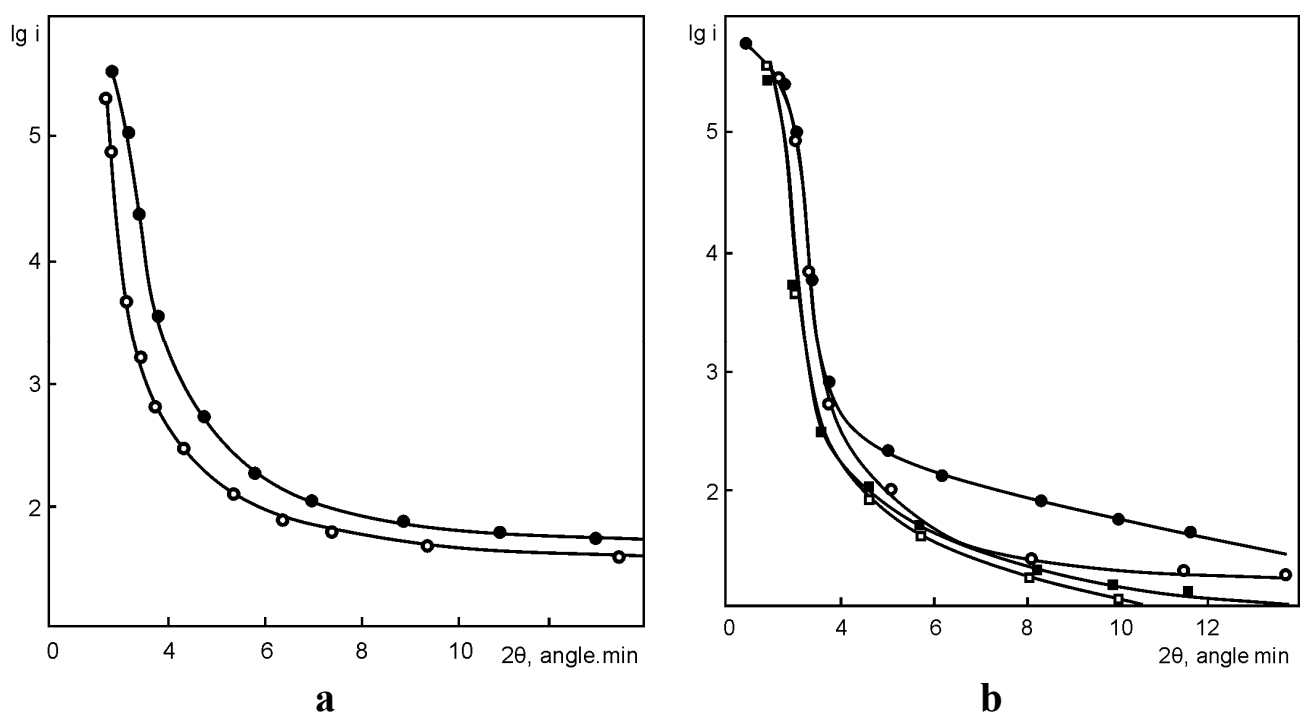

As shown in previous surveys, this tendency also appears to be typical for the films condensed under Earth-based conditions. However, films condensed in space appear to be more isotropic than the earth-based films, especially at comparatively large scattering angles. This observation fits with the conclusion that condensation under micro-gravity conditions leads to the formation of more equiaxial SMPs. Moreover, columnar allocation of structural elements, which affect the shape and alignment of the pores, is not observed.

The density values of the space- and Earth-based films determined by the decrease in monochromatic X-ray irradiation are similar to one another. The same is true for the range of 
thicknesses studied here: the total volumetric concentration of SMPs in space and earth-based films are similar; however, the largest SMPs account for a greater volume fraction in space-based films than in Earth-based films.

Thus, an array of experiments on orbital stations has shown that condensation under space conditions contributes to the formation of more equiaxial and larger SMPs in fine metal films than under Earth-based conditions; however no substantial difference was observed between the total SMP quantities of space and Earth-based films.

\section{Conclusions}

Mechanisms of pore formation in metal coatings condensed in Earth-based and space conditions are similar, although they have particular features, occurring due to space microgravity. As to total porosity level, no substantial difference between space and Earth-based condensates has been detected. Due to weakening of molecular flow orienting impact in space conditions, cosmic films reveal no columnar structure and SMPs, orienting in the molecular flow direction. The distribution of SMPs as regards size is more uniform, their dispersion level is much lower compared to the Earth-based samples, while the shape of SMPs appears to be close to equiaxial. This might be caused as a result of the amplification of the porosity diffusion-vacancy mechanism due to weakening of the role of adsorption processes.

These experiments were conducted on films of $\mathrm{Cu}$ condensed on the OS "SALUT-6" by the apparatus "ISPARITEL M" (Figure 4). Evaporable material was heated in a molybdenic bowl by means of electron bombardment [6]. The evaporable unit (Figure 4a) together with the bowl and supports, was installed in the sluice chamber, which could be connected to the outboard area upon depressurisation. Therefore, the composition of residual gases in the sluice chamber was affected by the composition of the atmosphere near the orbital station. Supports were fixed in a special drum (Figure $4 \mathrm{~b}$ ) that was rotated, contributing to their replacement during the experiment. A shutter, which separates the evaporator and the drum, prevents the samples from being affected by the fumes of the evaporant until complete heating of the bowl and provides partial execution of preset evaporation time (Figure 4d). Condensation was conducted on non-heated supports made of glass, titanium (both polished and non-polished), lacquered titanium and black-reinforced plastic. The duration of condensation was varied from 1-200 s, allowing for formation and investigation of films with thicknesses ranging from several nanometres to micrometres. In some cases, the total condensation time was 600-750 s due to the redeposition of the material on its own sub-layer.

Another set of tests was conducted on board the "MIR" orbital station. The condensation of coatings of $\mathrm{Cu}$ with thickness up to $1 \mu \mathrm{m}$ was tested on a static and movable tape drive within a thin polyimide support. These experiments were also repeated under similar conditions on Earth, which excluded the influence of microgravity.

Small-angle X-ray scattering (SAXS) is very effective for characterization of the submicroporosity of substances [7]. Pores, as local electron density inhomogeneities, cause diffuse SAXS, the equivalent scattering particle pores being identical in size, shape, and electron density drop. 
Figure 4. The apparatus "ISPARITEL M": (a) two monoblocks each of which has its electron beam gun for thin metal coating plating; (b) six-position drum with two cassettes with samples (c) on each face; (d) the shutter.

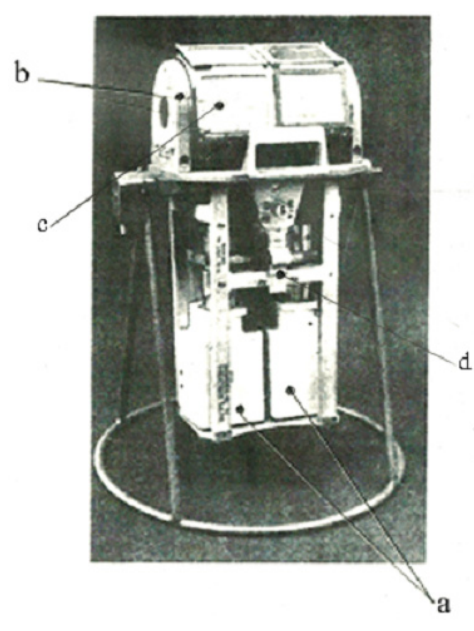

SAXS was conducted according to the previously described technique [7,8]. The SAXS indicatrixes were registered by a small-angle X-ray diffractometer using slit-like collimation of the primary MoK $\alpha$ X-ray beam. During treatment, the SAXS intensity for each scattering angle was transformed to a point-like collimation. The absolute SAXS intensity was measured against a calibrated standard according to the method of Kratky [8]. For each X-ray measurement, the small-angle background of the diffractometer was also calibrated. The reproducibility error of this curve in the range of scattering angles investigated here, as well as that of the SAXS experimental indicatrixes, was in the range of $1.5 \%-2 \%$.

The morphology, concentration, and size distribution of submicropores in $\mathrm{Cu}$ coatings were investigated by SAXS. We analyzed the following values [9]: type of angular distribution; asymptotics and the integral parameters of the dispersion indices of SAXS related to the typical size:

$$
l_{n}=1 / 2 \pi \int_{0}^{\infty} \tilde{I}(q) q \mathrm{~d} q
$$

the typical volume :

$$
V_{n}=\tilde{I}(0)
$$

where: $\tilde{I}(q)=I(q) ; I(q)$, SAXS intensity; $q$, diffraction vector; $P=1 / 2 \pi^{2} \int_{0}^{\infty} \tilde{I}(q) q^{2} \mathrm{~d} q$, invariant indicatrix SAXS; and the typical SMP shadow area:

$$
f_{n}=1 / 2 \pi \int_{0}^{\infty} \tilde{I}(q) q \mathrm{~d} q
$$

Investigations by the method of absorption of monochromatic X-ray radiation were conducted according to the technique described in [7].

The porosity of $\mathrm{Cu}$ coatings was measured and calculated according to the previously described method for Al coatings of Ar low-temperature desorption [10]. 


\section{References}

1. Cheremskoy, P.; Slezov, V.; Betechtin, V. Pores in Solid State; Energoatomizdat: Moscow, Russia, 1990; p. 376.

2. Skatkov, L.; Cheremskoy, P. Phase state and thermodynamical principles in porous systems. J. Mat. Sci. Eng. 2011, 5, 478-487.

3. Skatkov, L.; Gomozov, V. About the Pore Formation in Nanocrystal Metal Films. Proceeding of Advanced Processing for Novel Functional Materials, Dresden, Germany, January 2008; pp. 495-499.

4. Skatkov, L. Advances in Sintering Science and Technology; Bordia, R., Olevsky, E., Eds.; American Ceramic Society: Hoboken, NJ, USA, 2010; Volume 209, pp. 429-436.

5. Cheremskoy, P.; Pugachov, A.; Sobol, O.; Malyhin, S.; Toptygin, A.; Panikarsky, A. Condensation-dependent porosity in film nanostructures. Funct. Mat. 2005, 12, 539-547.

6. Palatnik, L.; Toptygin, A.; Cheremskoy, P. Space: Materials, Science and Engineering; NANU Publisher House: Kiev, Ukraine, 2000, p. 312.

7. Cheremskoy, P. Methods of Investigation on Solid State Porosity; Energoatomizdat: Moscow, Russia, 1985; p. 110.

8. Glater, O.; Kratky, O. Small Angle X-Ray Scattering; Academic Press: London, NY, USA; 1982.

9. Skatkov, L.; Gomozov, V.; Bayrachiy, B. Investigation of porosity and fractal properties of the pyrolytic $\mathrm{MnO}_{2}$ films in the capacitor structure. J. Anal. Appl. Pyrol. 2012, 98, 247-249.

10. Skatkov, L.; Cheremskoy, P.; Gomozov, V.; Bayrachniy, B.; Tulskiy, G.; Deribo, S. The influence of space environment on substructure of light-absorbing thermoregulating Al coatings. Coatings 2011, 1, 108-116.

(C) 2012 by the authors; licensee MDPI, Basel, Switzerland. This article is an open access article distributed under the terms and conditions of the Creative Commons Attribution license (http://creativecommons.org/licenses/by/3.0/). 